\title{
Plankton and Macrobiota Composition and Diversity of Three Tropical Freshwaters Rivers in Ogun and Ondo States, Southwest Nigeria
}

\author{
Taofikat Abosede ADESALU ${ }^{1 *}$, Olakunbi Anike KUNRUNMI ${ }^{1}$, \\ Muyideen Owonire LAWAL ${ }^{2}$
}

\author{
IUniversity ofLagos, Faculty of Science,Department of Botany,Akoka, Nigeria; tadesalu@unilag.edu.ng("correspondingauthor); kunbikunrunmi@yahoo.com \\ ${ }^{2}$ University of Lagos, Faculty of Science, Department of Marine Sciences, Akoka, Nigeria; lawdeen2003@yahoo.com
}

\begin{abstract}
Three different rivers in Ogun and Ondo states were investigated for both micro and macro-biota of the water bodies. Several physical and chemical properties of these rivers were determined. The $\mathrm{pH}$ value of the studied water bodies was essentially neutral with salinity values between 0.02 - 4.0\% . Microalgae communities were represented by three divisions: Cyanophyta, Bacillariophyta and Chlorophyta at Oluwa and Ifara Rivers (Ondo state), while at Ibefun River (Ogun state), five divisions: Cyanophyta, Bacillariophyta, Chlorophyta, Euglenophyta and Pyrrhophyta were identified. Diatoms dominated these water bodies, with Navicula radiosa Kutz. at Ifara River, Fragilaria sp. in Oluwa River, while out of 90 algal taxa identified in Ibefun river, 64 were diatoms species belonging to 26 genera, with Melosira sp. and Synedra sp. recording the highest numbers of cell count. Dinoflagellates recorded only Peridinium sp. while Phacus orbicularis Hubner and Trachelomonas sp. dominated the euglenoids. For the zooplankton composition at Ibefun, rotifers (75.95\%) were represented by Brachionus sp., which recorded 62.03\%, and Gastropus sp. with 13.92\% of the total zooplankton, thus dominated the spectrum, while the copepod recorded $22.78 \%$ of the total organisms, with Copilia sp. and Euchirella sp. having $8.86 \%$ each. The macrobenthic invertebrates were represented by 3 taxa, belonging to 3 groups, with the dominant group Insecta accounted for $57 \%$ of the total individuals ( 7 individuals $/ \mathrm{m}^{2}$ ), while Oligochaeta and Hirudinea accounted for $29 \%$ and $14 \%$ respectively of the total individuals at Oluwa and Ifara Rivers. At Ibefun River, the macrobenthic invertebrates were represented by 5 taxa, belonging to 3 groups, Bivalves, Oligochaeta and Insecta, with bivalves being the dominant group $\left(51.7 \%\right.$ of the total individuals, as 64 individuals $\left./ \mathrm{m}^{2}\right)$, while Oligochaeta and Insecta accounted for $26.6 \%$ and $21.9 \%$ respectively of the total individuals. The dominant taxon, Macoma cumana, accounted for 42.2\%. A total of seventeen (17) finfish and shellfish species comprised of thirteen (13) finfish and four (4) shellfish species were recorded, being representative of freshwater and brackish water species; Clarias anguillaris (Clariidae), Tilapia zilli (Cichlidae), Chrysichthys nigrodigitatus (Bagridae), Alestes spp. and Macrobrachium vollenhovenii (Palaemonidae) were the most common species observed.
\end{abstract}

Keywords: algae, benthos, fish, plankton, rivers, water chemistry

\section{Introduction}

Algae are one of the most important primary producers in almost every aquatic ecosystem. They are not only the nutrition source of themselves and the other living organisms in water, they also cause increases in the dissolved oxygen rate. That is why phytoplankton is greatly affected by environmental pollution and changes in the aquatic ecosystem. Plankton plays a major role in the determination of the biological productivity of water bodies. It represents a common component in ponds and lakes of various sizes, rivers, estuaries and world oceans. Plankton is a major food source associated with numerous fauna in these aquatic habitats which they in turn are linked to other predators, including those leading to the higher trophic levels. Through the process of photosynthesis, they are capable of harvesting solar energy in their transformation of basic substances in the water, being able to multiply and thus represent a food and energy product for various animal species.
In addition, a major bi-product of their photosynthesis is oxygen, which is released into the water as another essential commodity for biota in these habitats. Like all plants, phytoplankton has the ability to fix carbon by photosynthesis (Nwankwo, 1996). Reports from Boney (1989) described phytoplankton as being predominantly autotrophs and therefore form the base of the food chain in an aquatic environment. They perform more than $95 \%$ of photosynthesis in the ocean, which amounts to nearly half of the world's primary production and are responsible for the release of nearly half the oxygen in the atmosphere (Castro and Huber, 2005).

They are used as indicators of environmental conditions within the aquatic environment because their populations are especially sensitive to changes in nutrient levels and other water quality conditions. Ecologically, algae are the widespread of the photosynthetic plants, forming bulk of the carbon assimilating, floating microscopic cells in the sea and fresh water. Macrobenthic invertebrates are useful bio-indicators providing 
a more accurate understanding of changing aquatic conditions than chemical and microbiological data, which at least give short-term fluctuations (Ravera, 2000; Ikomi et al., 2005). The composition, abundance and distribution of benthic macroinvertebrates can be influenced by water quality and differences in the local environmental conditions (Haslam, 1990; Odiete, 1999). Chironomidae and lumbriculidae have been reported to dominate aquatic benthic invertebrates' communities (Victor and Ogbeibu, 1985; Hynes, 1998) as they hardly show any habitat restriction (Victor and Ogbeibu, 1991) and are known to replace other invertebrate taxa in streams perturbed by human activities.

Nigerian aquatic systems are subjected to pollution pressures associated with urbanization and population growth (Nkwoji et al., 2010). Many of the rivers and creeks are used for irrigation, flood control, fisheries development and provision of potable water to towns and the surrounding rural areas. Along the approximately $853 \mathrm{~km}$ coastline of Nigeria there are estuaries and lagoons receiving effluents from many rivers and creeks most of which are routes for the introduction of pollutants. Benthological studies in Nigeria include Ajao and Fagade (1990), Brown (2000) and Edokpayi et al. (2004), who have focused majorly on general species composition and distribution.

Therefore, the authors consider that this study will contribute greatly to the freshwater microalgae, macro invertebrates and fishes database of the study sites. In this regard, the study aimed to make a contribution to the knowledge of the diversity of microalgae, macro invertebrates and fish communities in these rivers.

\section{Materials and Methods}

\section{Description of study sites}

Ondo state: The stations are within Omotosho/Agodo power plant transmission line which covers a distance of $50 \mathrm{~km}$ (from Omotosho Power station in Ondostate to Agodo, Ogun waterside, Ogun state). The Omotosho/Agodo power plant transmission line passes through the communities like Faji, Oloruntedo (where the transmission line tease off to Agodo), Agirifon, Araromi, Ilusin and Agodo. The vegetation along these communities includes Cola nitida, Ficus sur, Elaeis guineesis, Anthocleista vogelii and Hevea brasiliensis (Plantation). Two shallow and rocky rivers (Oluwa and Ifara) were analysed for their biota in this state. Three sampling stations were chosen for each of the two rivers, with Oluwa River having Station $1\left(\mathrm{~N}^{\circ} 6^{\circ}\right.$ $43^{\prime} 16.8^{\prime \prime} \mathrm{E} 004^{\circ} 42^{\prime} 49.1^{\prime \prime}$ ), Station 2 (N06 43'53.8"E004

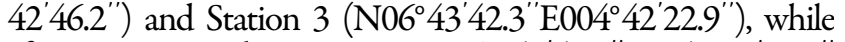
Ifara River coordinates were N06 43'41.3"E004 $37^{\prime} 00.5^{\prime \prime}$

Table 1. Coordinates of different stations at Ibefun

\begin{tabular}{|c|c|c|}
\hline Station & Latitude & Longitude \\
\hline 1 & N06 $6^{0} 37.225^{\prime}$ & $\mathrm{E} 003^{0} 48.442^{\prime}$ \\
\hline 2 & N06 $6^{0} 37.604^{\prime}$ & $\mathrm{E} 003^{0} 48.233^{\prime}$ \\
\hline 3 & N06 $6^{0} 37.964^{\prime}$ & $\mathrm{E} 003^{0} 48.011^{\prime}$ \\
\hline 4 & $\mathrm{~N}^{0} 6^{0} 38.380^{\prime}$ & $\mathrm{E} 003^{0} 47.936^{\prime}$ \\
\hline 5 & N0 $6^{0} 38.700^{\prime}$ & $\mathrm{E} 003^{0} 47.812^{\prime}$ \\
\hline 6 & N06 $6^{0} 39.095^{\prime}$ & $\mathrm{E} 003^{\circ} 47.792^{\prime}$ \\
\hline 7 & N0 $6^{0} 39.226^{\prime}$ & $\mathrm{E} 003^{0} 47.457^{\prime}$ \\
\hline 8 & N0 $6^{0} 39.340^{\prime}$ & $\mathrm{E} 003^{0} 46.647^{\prime}$ \\
\hline 9 & N06 $6^{0} 39.796^{\prime}$ & $\mathrm{E} 003^{0} 46.425^{\prime}$ \\
\hline 10 & N0 $6^{0} 40.412^{\prime}$ & $\mathrm{E} 003^{0} 46.495^{\prime}$ \\
\hline
\end{tabular}

(Station 1), N06 43'30.7'E004' 36' 56.3" (Station 2) and N06 $43^{\prime} 13.3^{\prime \prime} \mathrm{E} 004^{\circ} 36^{\prime} 55.3^{\prime \prime}$ (Station 3).

Ibefun (Ogun state): Ibefun River falls within the lowland rainforest zone where there is high humidity and predominance of herbs, shrubs and tree species. Generally, the ecosystems encountered included secondary regenerating forests and freshwater swamp. Within these, bush fallow of different ages and farmlands were also recorded in the area. The most conspicuous economic plants observed were Cola spp. (Kolanut), Discorea spp. (Yam), Elaeis guineensis (Oil Palm), Mangifera indica (Mango), Musa spp. (Plantain and Banana), Manihot esculenta (Cassava), Theoborma cacao (Cocoa), Zea mays (Maize), Mangifera indica (Mango), Anacardium occidentale (Cashew), Rauvolfia vomitoria (Swizzle stick), Colocasia esculenta (Cocoyam), Talinum triangulare (Water leaf) among others. The study site has riparian vegetation and freshwater swamp vegetation like Raphia hookeri and macrophytes like Eichorrnia crassipes (Mart) Solm. and Ceratophyllum demersum. Ten sampling stations were chosen to reflect the differences in biological characteristics which exist in the same body of water using Global Positioning System (GPS) (Table 1). This river enters into the Lagos lagoon.

\section{Collection of biologicalsamples}

Plankton: For plankton collection, duplicate water samples were collected; surface water samples were collected directly into already labelled $500 \mathrm{ml}$ plastic bottles; plankton net was also towed at low speed for 5 minutes and both sample types $s$ were fixed with $4 \%$ unbuffered formalin. This method was used in order to have record of all plankton, including nanoplankton that may be present in water bodies. Samples were then taken into the laboratory where they were allowed to settle for $24 \mathrm{~h}$ and then decanted to a known volume in appropriately labelled plastic containers. In the laboratory, the plankton samples were concentrated to $10 \mathrm{ml}$.

For analysis, five drops of each concentrated sample $(10 \mathrm{ml})$ were investigated at different magnifications (X100 and X400) using Olympus XSZ-N107 photomicroscope. Taxonomic keys employed in the identification of plankton taxa included Hustedt (1930-1937), Atrick and Reimer (1966), Whitford and Schumacher (1973), Krammer and Large-Bertalot (1986), Patrick and Reimer (1975), Prescott (1962), Wimpenny (1966), Olaniyan (1975), Nwankwo (1984), Davis (1955), Desikachary (1959), Wiafe and Frid (2001).

Macro invertebrates: Benthos sampling was carried out with Van Veen grab of sediments, except for some locations where direct collection of sediments was made. Each location was geo- referenced using geographic position system. Grab samples and direct sediments samples were initially washed on deck through sieves of $0.5 \mathrm{~mm}$ mesh and the retained materials were preserved in $4 \%$ formalin for further sorting in the laboratory. The identification of the species was according to taxonomic keys provided in Quigley (1977), Atobatele et al. (2005) and Ibemenuga and Inyang (2006).

Fish and Fisheries study: The investigation of fish and fisheries of the study area was carried out based on oral interview of the local fishermen, physical observation of the fishing methods and processing of landings. Some of the fishing gear 
248

used in the study area was gillnet, cast net and fish trap. The finfish and shellfish recorded in the study area included both freshwater and brackish water species. Fish samples were bought from the fishermen and then taken to the laboratory for further processing. The condition factor $(\mathrm{K})$ was determined using Bannister (1976) method.

\section{Physico-chemical analysis}

Surface water samples were analyzed in-situ for some of the parameters. The $\mathrm{pH}$ was determined with Electronic Cole-Parmer Testr3, while the salinity was measured using a handheld refractometer. Dissolved oxygen and chemical oxygen demand were determined using titrimetric method. Winkler's method was applied for the determination of biological oxygen demand.

\section{Statistical analysis}

To obtain the estimates of species diversity, three community structure indices Margalefs diversity index (d), Shannon-Weaner index $\left(\mathrm{H}^{1}\right)$ (Shannon and Weaver, 1963) and Species equitability (j) or Evenness (Pielou, 1975) were used. For benthic analysis, bio-statistical methods used were according to Ogbeibu (2005).

\section{Results}

\section{Physico-chemical analysis}

The $\mathrm{pH}$ value ranged between 7.07 and 7.54 for the sampled stations, while the salinity was between $0.02-0.03 \%$. The sulphates, phosphate-phosphorus and nitrate-nitrogen ranged between 2.0 and 3.0, 0.003 and $0.005,0.138$ and $0.210 \mathrm{mg} / \mathrm{L}$ respectively. Dissolved oxygen value was high, with $5.1 \mathrm{mg} / \mathrm{L}$ and $4.5 \mathrm{mg} / \mathrm{L}$ recorded, while biological oxygen demand and chemical oxygen demand ranged between $4.0-6.0 \mathrm{mg} / \mathrm{L}$ for the sites.

\section{Plankton}

Microalgae at Ifara and Oluwa Rivers: Three classesBacillariophyceae, Cyanophyceae and Chlorophyceae were recorded for both rivers. Diatoms dominated both samples, with frequent taxa being Navicula radiosa Kutz. at Ifara River and Oluwa River, whereas Fragilaria sp. recorded the highest frequency; blue green algae recorded Oscillatoria sp. which was rear in the samples and Stigeoclonium ably represented the green algae (Table 2).

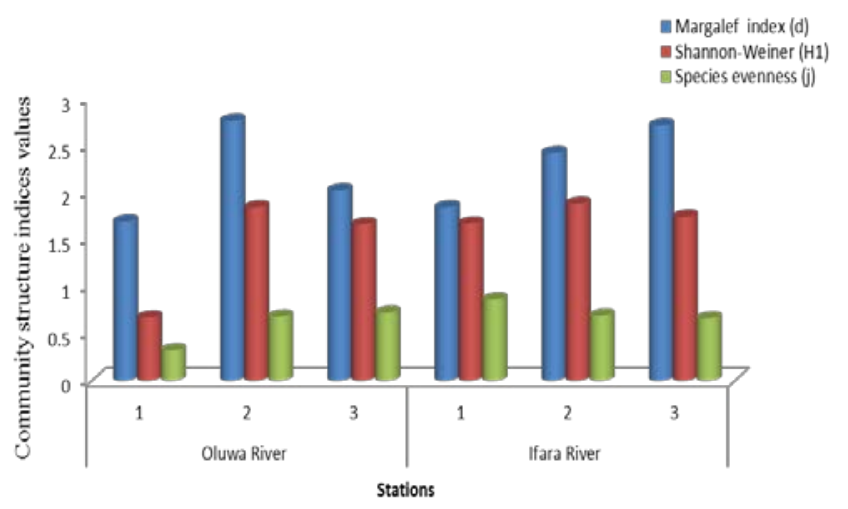

Fig. 1. Comparison of microalgal community structure indices at Oluwa and Ifara Rivers, Ondo State
Community structure analysis recorded highest species evenness (0.86 and 0.72) at Stations 1 and 3 in Ifara and Oluwa Rivers respectively, while the lowest diversity value (1.69) was recorded at Station 1 in Oluwa River (Fig. 1). Zooplankton samples were not diverse, as only two species were recorded for the sample.

\section{Microalgae at Ibefun River}

The total microalgae (cells count) and species composition is presented in Table 3. Five different divisions- Bacillariophyta, Chlorophyta, Cyanophyta, Euglenophyta and Pyrrophyta were represented. Out of the 90 algal taxa identified, 64 were diatoms species, belonging to 26 genera, with Melosira sp. and Synedra sp. recording the highest number of cell count. The green algae were represented by many species out of which Crucigenia irregularis Wille, Desmodesmus abundans var. brevicauda G. M Smith and Scenedesmus quadricauda var. maxima W\&G.S.West were frequently encountered. The cyanophyta was ably represented by five genera, with Spirulina sp. being the most frequent species. Dinoflagellates recorded only Peridinium sp., while Phacus orbicularis Hubner and Trachelomonas sp. dominated the euglenoids (Table 3). Community structure analysis recorded highest value (0.91) of species evenness (j) at Stations 9 and 10, while the lowest diversity value (4.67) was recorded at Station 4, whereas Shannon-Weaner index highest value (3.17) corresponded to highest species evenness (j) at Station 9 (Fig. 2).

\section{Zooplankton}

The zooplankton composition at Ibefun was dominated by rotifers (75.95\%) represented by Brachionus sp. which recorded $62.03 \%$ and Gastropus sp. with $13.92 \%$ of the total zooplankton observed. The copepod recorded $22.78 \%$ of the total organisms, with Copilia sp. and Euchirella sp. having $8.86 \%$ each, while Scolecithrix sp. had $5.06 \%$. The lowest percentage occurrence $(1.27 \%)$ was recorded by Brachyuran sp. (Decapoda) (Table 4). The presence of Copillia and Euchirella spp. in zooplankton composition might be an indicator of sea water incursion to the Ibefun River. The community structure analysis as compared with that of microalgae (Fig. 4) showed a diverse ecosystem in terms of microalgae which invariably supports the growth of few species of zooplankton recorded hereby, showing in the species evenness that slightly followed the same pattern in plankton (Fig. 3).

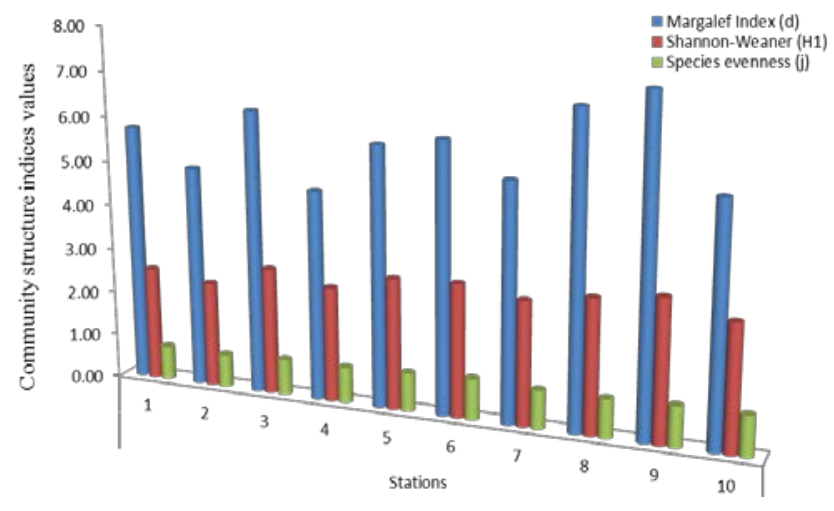

Fig. 2. Comparison of microalgal community structure indices at Ibefun River, Ogun State 
Table 2. Microalgae composition and abundance $(\mathrm{cell} / \mathrm{s} / \mathrm{mL})$ at Oluwa and Ifara Rivers, Ondo state

\begin{tabular}{|c|c|c|c|c|c|c|}
\hline \multirow{3}{*}{ Microalge composition } & \multicolumn{3}{|c|}{ Oluwa River } & \multicolumn{3}{|c|}{ Ifara River } \\
\hline & \multicolumn{3}{|c|}{ Stations } & \multicolumn{3}{|c|}{ Stations } \\
\hline & 1 & 2 & 3 & 1 & 2 & 3 \\
\hline \multicolumn{7}{|l|}{ Division: Bacillariophyta } \\
\hline \multicolumn{7}{|l|}{ Class: Bacillariophyceae } \\
\hline \multicolumn{7}{|l|}{ Order: Achnanthales } \\
\hline Cocconeis scutelum Ehr. & - & 3 & 1 & _- & _- & 1 \\
\hline \multicolumn{7}{|l|}{ Order: Coscinodiscales } \\
\hline Coscinodiscus sp. & & & & _ & 3 & _- \\
\hline \multicolumn{7}{|l|}{ Order: Thallassiophysales } \\
\hline Amphora sp. & _- & 2 & _ & _ & 3 & 1 \\
\hline \multicolumn{7}{|l|}{ Order: Cymbellales } \\
\hline Cymbella sp. & _- & - & 1 & & & \\
\hline Gomphonema angustum Agardh & $\overline{1}$ & - & - & 3 & 36 & - \\
\hline G. clavatum Ehrenberg & - & 1 & - & 1 & - & - \\
\hline G. gracile Ehr. & & & & - & 46 & - \\
\hline Gomphonema sp. & - & 1 & _- & & & \\
\hline \multicolumn{7}{|l|}{ Order: Eunotiales } \\
\hline Eunotia sp. & . & 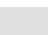 & . & _ & 1 & - \\
\hline \multicolumn{7}{|l|}{ Order: Fragillariales } \\
\hline Fragilaria capucina Desmarziers & - & - & 1 & 4 & 1 & - \\
\hline Fragilaria sp. & 54 & 50 & _- & 5 & - & 32 \\
\hline Frustulia vulgaris Thwaites & & & & - & - & 2 \\
\hline Surirella sp. & & & & - & - & 1 \\
\hline Synedra sp. & 3 & 19 & 3 & - & $\overline{93}$ & 42 \\
\hline Terpsinoe americana (Bail.) Ralfs & & & 18 & - & _- & 2 \\
\hline Ulnaria ulna (Nitzsch.) Ehrenberg & & & & - & 17 & 1 \\
\hline \multicolumn{7}{|l|}{ Order: Tabellariales } \\
\hline Tabellaria fenestrata (Lyng.) Kutzing & _- & 2 & - & _- & 2 & 22 \\
\hline Order: Mastogloiales & & & & & & \\
\hline Mastogloia sp. & - & - & 2 & 1 & - & - \\
\hline Meridion sp. & $\overline{1}$ & - & _- & & & \\
\hline Order: Naviculales & & & & & & \\
\hline Diatoma sp. & & & & _- & _- & 1 \\
\hline Navicula cryptocephala Kutz. & & & & - & $\overline{1}$ & - \\
\hline Navicula decussis Oestrup & - & 1 & _- & & & \\
\hline N. exigua (Greg.) O. Muller & $\overline{1}$ & 2 & - & & & \\
\hline Navicula mutica Kutzing & 1 & - & - & & & \\
\hline N. radiosa Kutz. & 1 & $\overline{10}$ & $\overline{7}$ & 10 & 78 & 12 \\
\hline Neidium sp. & - & 1 & _- & & & \\
\hline Pinnularia abaujensis (Plant) Ross & & & & - & 3 & _- \\
\hline P. legumen Ehr. & & & & - & 1 & - \\
\hline P. maior Kutzing & & & 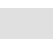 & - & - & $\overline{1}$ \\
\hline Order: Nitzschiales & & & & & & \\
\hline Nitzschia sublinearis Hustedt & - & 1 & _- & & . & \\
\hline Pinnularia sp. & $\overline{1}$ & - & - & _- & - & 2 \\
\hline Division: Cyanophyta & & & & & & \\
\hline Class: Cyanophyceae & & & & & & \\
\hline Order: Hormogonales & & & & & & \\
\hline Chroococcus sp. & _- & _- & 22 & & & \\
\hline Oscillatoria proboscidea Gom. & - & 20 & - & & & \\
\hline Oscillatoria sp. & - & 45 & - & 2 & & \\
\hline Spirulina sp. & & & & _- & 1 & _- \\
\hline Division: Chlorophyta & & & & & & \\
\hline Class: Chlorophyceae & & & & & - & _- \\
\hline Order: & & & & & & \\
\hline Closterium moniliferum Ehrenb. & - & - & 1 & & & \\
\hline Cosmarium sp. & - & $\overline{1}$ & & & & \\
\hline Stigeoclonium sp. & - & - & 30 & _ & 42 & 1 \\
\hline Total number of species & $\overline{8}$ & $\overline{15}$ & 10 & $\overline{7}$ & 15 & 14 \\
\hline Total number of individuals & 63 & 159 & 86 & 26 & 328 & 121 \\
\hline Margalef index (d) & 1.69 & 2.76 & 2.02 & 1.84 & 2.42 & 2.71 \\
\hline Shannon-Weaver $\left(\mathrm{H}^{1}\right)$ & 0.67 & 1.84 & 1.66 & 1.67 & 1.88 & 1.74 \\
\hline Species evenness ( $j$ ) & 0.32 & 0.68 & 0.72 & 0.86 & 0.69 & 0.66 \\
\hline
\end{tabular}

\section{Macrobenthic invertebrates}

Oluwa and Ifara Rivers: The macrobenthic invertebrates were represented by 3 taxa belonging to 3 groups (Table 5). The dominant group was the Insecta, accounting for $57 \%$ of the total individuals (7 individuals $/ \mathrm{m}^{2}$ ), while Oligochaeta and Hirudinea accounted for 29\% and 14\% respectively of the total individuals. The dominant taxon was Chironomus spp. accounting for 57\%, while Glossiphonia spp. was the least represented, with $14 \%$ of the total individuals (7 individuals $\left./ \mathrm{m}^{2}\right)$ recorded. The general diversity $\left(\mathrm{H}^{1}\right)$ and species richness (d) were the highest at Stations 1 and 2 with corresponding values of 0.30 and 1.45 respectively (Fig. 4). At Station 3, the lowest values of 0.28 and 0.91 were recorded for the diversity and species richness respectively.

Ibefun River: The macrobenthic invertebrates were represented by 5 taxa belonging to 3 groups (Table 6). Bivalve was the dominant group accounting for $51.7 \%$ of the total individuals (64 individuals $/ \mathrm{m}^{2}$ ), while Oligochaeta and Insecta accounted for $26.6 \%$ and $21.9 \%$ respectively of the total individuals. The dominant taxon, Macoma cumana, accounted 
Table 3. Microalgae composition and abundance (cells $/ \mathrm{mL}$ ) at Ibefun River (Ogun state)

\begin{tabular}{|c|c|c|c|c|c|c|c|c|c|c|c|}
\hline & \multirow{2}{*}{ Microalge composition } & \multicolumn{10}{|c|}{ Stations } \\
\hline & & 1 & 2 & 3 & 4 & 5 & 6 & 7 & 8 & 9 & 10 \\
\hline & \multicolumn{11}{|l|}{ Division: Bacillariophyta } \\
\hline & \multicolumn{11}{|l|}{ Class: Bacillariophyceae } \\
\hline 1 & Achanthes sp. & - & - & - & - & - & _- & - & - & - & 3 \\
\hline 2 & Amphora coffeaeformis (Agardh) Kutzing & - & - & - & - & - & - & - & - & 1 & - \\
\hline 3 & A. holstica Hustedt & 1 & - & - & - & - & _- & - & - & - & - \\
\hline 4 & Amphora sp. & _- & - & 3 & _- & _- & _ & _- & _- & 4 & _ \\
\hline 5 & Asterionella formosa Hassal & - & - & - & - & - & _- & - & - & - & 1 \\
\hline 6 & Bacillaria paxillifera (O.F. Muller) Hendey & 2 & 2 & - & - & - & _- & - & 5 & - & 6 \\
\hline 7 & Cocconeis scutellum Ehr. & 2 & 1 & _ & - & 4 & _ & _- & 1 & 3 & 3 \\
\hline 8 & Cyclotella sp. & - & - & 6 & 1 & 4 & 1 & 6 & 10 & 1 & 1 \\
\hline 9 & Cymbella affinis Kutzing & - & - & - & - & - & - & 1 & - & - & - \\
\hline 10 & C. brehmii Hustedt & - & - & - & - & 2 & - & - & - & - & _ \\
\hline 11 & C. prostrata (Berkely) Cleve & 2 & _- & - & - & _- & _- & - & - & - & _ \\
\hline 12 & Cymbella sp. & - & - & - & - & - & _- & - & - & - & 1 \\
\hline 13 & Diatomella balfouriana Greville & 4 & - & 2 & - & - & - & 3 & 1 & - & - \\
\hline 14 & Eunotia sp. & - & - & - & - & 1 & - & - & _ & - & - \\
\hline 15 & Fragilaria capucina Desmarziers & - & _- & - & _- & _- & _ & _- & _- & 1 & _ \\
\hline 16 & F. crotonensis Kitton & - & - & - & - & - & 4 & - & - & 2 & - \\
\hline 17 & Fragilaria sp. & 30 & - & - & - & - & - & - & 5 & - & - \\
\hline 18 & Frustulia nobilis Schumard & - & - & - & - & - & - & - & - & - & 2 \\
\hline 19 & F. rhomboids (Ehr.) de Toni & _ & _- & - & _- & 1 & _- & _- & _- & _- & _ \\
\hline 20 & F. vulgaris Thwaites & - & - & - & - & - & _- & - & - & - & 1 \\
\hline 21 & Gomphonema angustum Agardh. & - & - & - & - & - & - & - & - & 2 & 1 \\
\hline 22 & G. clavatum Ehrenberg & - & - & - & - & 1 & - & - & 1 & - & - \\
\hline 23 & G. gracile Ehrenberg & - & - & - & - & - & _- & _- & - & 2 & 2 \\
\hline 24 & G. lanceolatum Ehr. & - & - & - & - & - & _- & - & - & 1 & _ \\
\hline 25 & G. subclavatum (Grunow) & 1 & - & - & - & - & - & - & 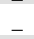 & 1 & - \\
\hline 26 & Gomphonema sp. & 2 & - & 1 & - & - & _- & - & - & - & - \\
\hline 27 & Gyrosigma acuminatum (Kutzing) Rabenhorst & - & - & - & - & - & - & - & - & 1 & $\overline{5}$ \\
\hline 28 & G. nodiferum (Grunow) Reimer & - & - & 1 & - & - & _ & - & - & - & - \\
\hline 29 & G. obscurum (W.Smith) Griffith \&Henfrey & - & - & - & - & - & - & - & 1 & 2 & - \\
\hline 30 & G. scalproides (Rabenhorst) Cleve & 1 & - & - & - & - & - & 1 & - & 2 & 1 \\
\hline 31 & G. spencerii (Quekett) Griffith \&Henfrey & - & - & - & - & 3 & _ & _- & - & - & _ \\
\hline 32 & Mastogloia sp. & - & - & - & - & 1 & - & - & - & 1 & - \\
\hline 33 & Melosira sp. & 9 & 45 & 37 & 34 & 22 & 1 & 12 & 10 & - & - \\
\hline 34 & Navicula angusta Grunow & - & - & - & - & - & 2 & - & - & - & - \\
\hline 35 & N. contenta (Grunow) & - & 2 & - & - & - & - & - & - & - & - \\
\hline 36 & N. decussis Oestrup & - & - & 1 & - & - & _- & 1 & 1 & - & _ \\
\hline 37 & N. exigua (Greg) O. Muller & 2 & $\overline{2}$ & 1 & - & - & - & - & - & - & 2 \\
\hline 38 & N. muralis Grun & - & - & 1 & - & - & - & - & - & - & - \\
\hline 39 & N. mutica Kutzing & $\overline{1}$ & $\overline{6}$ & 1 & $\overline{3}$ & $\overline{4}$ & $\overline{1}$ & - & - & - & - \\
\hline 40 & N. radiosa (Kutz.) & 1 & - & 1 & 1 & - & 2 & 1 & 1 & 1 & 3 \\
\hline 41 & N. rhynchocephala Kutz. & - & - & 2 & - & - & _ & _- & - & - & _ \\
\hline 42 & Navicula sp. & 1 & - & 1 & 1 & - & - & 5 & 1 & 1 & 1 \\
\hline 43 & Neidium sp. & - & - & _- & - & $\overline{1}$ & - & 1 & - & - & _ \\
\hline 44 & Nitzschia acicularis W. Smith & 1 & 2 & 1 & - & 3 & 2 & - & 2 & 1 & - \\
\hline 45 & N. acuminate Gomont & - & 1 & _ & - & - & _- & - & - & - & - \\
\hline 46 & N. gracilis Hantzsch. & - & 1 & - & - & - & - & - & - & 1 & - \\
\hline 47 & N. ignorata Krasske & - & - & - & - & - & - & - & - & 1 & - \\
\hline 48 & N. obtuse W. Sm & - & 1 & - & - & - & 1 & - & 1 & - & - \\
\hline 49 & N.palea (Kutz) W.Sm & - & _- & 1 & - & - & _ & $\overline{2}$ & - & - & - \\
\hline 50 & N. sublinearis Hustedt & 3 & - & - & - & - & - & - & - & - & 1 \\
\hline 51 & N. sublitis Grun & - & $\overline{1}$ & - & - & - & - & $\overline{1}$ & - & - & _ \\
\hline 52 & Nitzschia sp. & 3 & - & 2 & - & 6 & - & 3 & 1 & 2 & - \\
\hline 53 & Pinnularia acrosphaeria Brebisson & - & - & _ & - & 1 & - & _- & - & - & - \\
\hline 54 & P. braunii (Grunow) Cleve & - & - & - & - & - & 1 & - & 2 & - & 5 \\
\hline 55 & P. gibba Ehrenberg & - & - & - & - & - & _ & - & - & 1 & _ \\
\hline 56 & P. stomatophora (Grunow) Cleve & - & - & - & - & - & - & 1 & 1 & - & - \\
\hline 57 & Pinnularia sp. & 1 & - & - & - & - & - & - & - & - & - \\
\hline 58 & Pleurosigma salinarum Grunow & - & - & - & - & - & - & 1 & - & - & - \\
\hline 59 & Stauroneis salina W. Smith & - & - & - & - & - & _- & - & 1 & - & - \\
\hline 60 & Synedra sp. & 14 & 14 & 12 & 11 & 9 & 2 & 10 & 9 & 10 & 1 \\
\hline 61 & Tabellaria fenestrate (Lyng.) Kutzing & 2 & 3 & 5 & 3 & _- & 1 & 1 & 5 & 3 & 2 \\
\hline 62 & Terpsinoe americana (Bail) Ralfs & - & - & - & - & - & _- & - & - & - & 8 \\
\hline 63 & Ulnaria ulna var. amphirynchus (Ehr.) Grun. & $\overline{1}$ & $\overline{1}$ & $\overline{4}$ & - & - & - & $\overline{3}$ & - & - & _ \\
\hline \multirow[t]{3}{*}{64} & U. ulna var. danica (Ehr.) van Huerck. & 5 & - & 4 & 4 & 4 & 1 & - & 1 & 1 & - \\
\hline & Division: Cyanophyta & & & & & & & & & & \\
\hline & Class: Cyanophyceae & & & & & & & & & & \\
\hline 65 & Chroococcus sp. & _- & 1 & 2 & 1 & 3 & _- & _ & _- & - & _ \\
\hline 66 & Oscillatoria acuminata Gomont & - & _- & 3 & 5 & 8 & 4 & - & - & 2 & - \\
\hline 67 & O. tenuis Ag. & 1 & - & _- & - & - & _- & - & - & - & - \\
\hline 68 & Phormidium sp. & - & 1 & - & - & 1 & - & - & 1 & - & - \\
\hline 69 & Polycystis sp. & 34 & - & - & - & - & - & - & - & - & - \\
\hline
\end{tabular}




\begin{tabular}{|c|c|c|c|c|c|c|c|c|c|c|c|}
\hline \multirow{3}{*}{70} & Spirulina sp. & 1 & 21 & 1 & 14 & 1 & 1 & _- & 2 & _ & - \\
\hline & \multicolumn{11}{|l|}{ Division: Chlorophyta } \\
\hline & Class: Chlorophyceae & & & & & & & & & & \\
\hline 71 & Chlorella sp. & _ & _- & 6 & 23 & _ & _ & - & _- & 6 & _- \\
\hline 72 & Chlorosarcina consociate (Klebs) G. M. Smith & - & - & - & - & - & - & - & $\overline{1}$ & _- & - \\
\hline 73 & Closterium abruptum (Lyngb.) Breb & _- & - & - & - & _- & & - & 1 & - & _- \\
\hline 74 & C. kutzingii f. sigmoides Irenee-Marie & 1 & 1 & 1 & 3 & 1 & 1 & 1 & 1 & - & 1 \\
\hline 75 & C. moniliferum Ehrenb. & _ & _ & _ & _ & _ & 1 & - & _ & _ & _ \\
\hline 76 & Cosmarium sp. & _- & 1 & 3 & 1 & - & _- & _- & _- & _- & _- \\
\hline 77 & Crucigenia irregularisWille & _- & _- & & 7 & 20 & 9 & 4 & - & 6 & - \\
\hline 78 & Desmodesmus abundans var. brevicauda G. M Smith & 3 & $\overline{1}$ & $\overline{7}$ & 8 & 3 & 5 & 5 & $\overline{1}$ & 2 & - \\
\hline 79 & Eudorina sp. & _ & 6 & 9 & 2 & 1 & 2 & - & _ & _ & _- \\
\hline 80 & Gonatozygon aculeatum Hastings & - & - & 1 & 3 & 2 & 1 & - & $\overline{1}$ & - & $\overline{1}$ \\
\hline 81 & Microsporafloccose (Vauch.) Lagerheim & _ & _- & _ & 1 & _ & _ & _ & _ & _- & _ \\
\hline 82 & Microspora sp. & 3 & - & - & - & - & 3 & 2 & - & 1 & - \\
\hline 83 & Pandorina sp. & _ & _- & _ & _- & _ & _ & _ & 1 & _- & _- \\
\hline \multirow[t]{3}{*}{84} & Scenedesmus quadricauda var. maxima $\mathrm{W} \& \mathrm{G}$.S. West & - & 3 & $\overline{4}$ & 4 & 10 & 2 & 5 & 1 & 1 & - \\
\hline & Division: Euglenophyta & & & & & & & & & & \\
\hline & Class: Euglenophyceae & & & & & & & & & & \\
\hline 85 & Euglena proxima Dang & _- & _- & 2 & _- & _- & _- & _- & _- & - & _- \\
\hline 86 & Euglena sp. & - & 2 & - & 2 & $\overline{7}$ & - & - & - & - & - \\
\hline 87 & Phacus orbicularis Hubner & - & 3 & 25 & 1 & 8 & $\overline{1}$ & $\overline{5}$ & $\overline{1}$ & - & - \\
\hline 88 & Trachelomonas sp. & $\overline{1}$ & 9 & 28 & 2 & 16 & 3 & 18 & 6 & 1 & - \\
\hline \multirow[t]{2}{*}{89} & Division: Pyrrhophyta & & & & & & & & & & \\
\hline & Class: Dinophyceae & & & & & & & & & & \\
\hline \multirow[t]{4}{*}{90} & Peridinium sp. & & & 9 & 2 & 1 & 2 & 1 & & 3 & \\
\hline & Total number of species & 29 & $\overline{25}$ & 34 & 24 & 30 & 25 & 25 & $\overline{31}$ & 32 & 22 \\
\hline & Total number of individuals & 133 & 131 & 188 & 137 & 149 & 54 & 94 & 77 & 68 & 52 \\
\hline & Margalef index (d) & 5.73 & 4.92 & 6.30 & 4.67 & 5.80 & 6.02 & 5.28 & 6.91 & 7.35 & 5.31 \\
\hline
\end{tabular}

Table 4. Zooplankton composition and abundance at Ibefun River (Ogun state) during sampling period

\begin{tabular}{|c|c|c|c|c|c|c|c|c|c|c|c|}
\hline & \multirow{2}{*}{ Microalge composition } & \multicolumn{10}{|c|}{ Stations } \\
\hline & & 1 & 2 & 3 & 4 & 5 & 6 & 7 & 8 & 9 & 10 \\
\hline & Rotifera & & & & & & & & & & \\
\hline 1 & Brachionus sp. & _- & _- & _ & _ & 3 & _- & 1 & 14 & 29 & 2 \\
\hline \multirow[t]{2}{*}{2} & Gastropus sp. & 3 & 2 & - & - & 3 & 2 & _ & _- & 1 & _- \\
\hline & Decapoda & & & & & & & & & & \\
\hline \multirow[t]{2}{*}{3} & Brachyuran sp. & _ & _- & 1 & _ & _- & _ & _- & _- & _- & - \\
\hline & Copepoda & & & & & & & & & & \\
\hline 4 & Copiliasp. & 2 & 1 & 1 & 1 & 1 & 1 & _ & _ & _- & _- \\
\hline 5 & Euchirella sp. & 1 & 1 & _- & _- & _- & _- & 3 & 1 & - & 1 \\
\hline \multirow[t]{6}{*}{6} & Scolecithrix sp. & _- & _- & $\overline{1}$ & $\overline{1}$ & $\overline{2}$ & - & _ & _- & - & - \\
\hline & Total number of species & $\overline{3}$ & 3 & 3 & 2 & 4 & $\overline{2}$ & $\overline{2}$ & $\overline{2}$ & 2 & $\overline{2}$ \\
\hline & Total number of individuals & 6 & 4 & 3 & 2 & 9 & 3 & 4 & 15 & 30 & 3 \\
\hline & Margalef index & 1.12 & 1.44 & 1.82 & 1.44 & 1.37 & 0.91 & 0.72 & 0.37 & 0.29 & 0.91 \\
\hline & Shannon-Weaner $\left(\mathrm{H}^{1}\right)$ & 1.01 & 1.04 & 1.1 & 0.69 & 1.31 & 0.64 & 0.56 & 0.25 & 0.15 & 0.64 \\
\hline & Species evenness $(\mathrm{j})$ & 0.92 & 0.95 & 1.00 & 1.00 & 0.94 & 0.92 & 0.81 & 0.36 & 0.22 & 0.92 \\
\hline
\end{tabular}

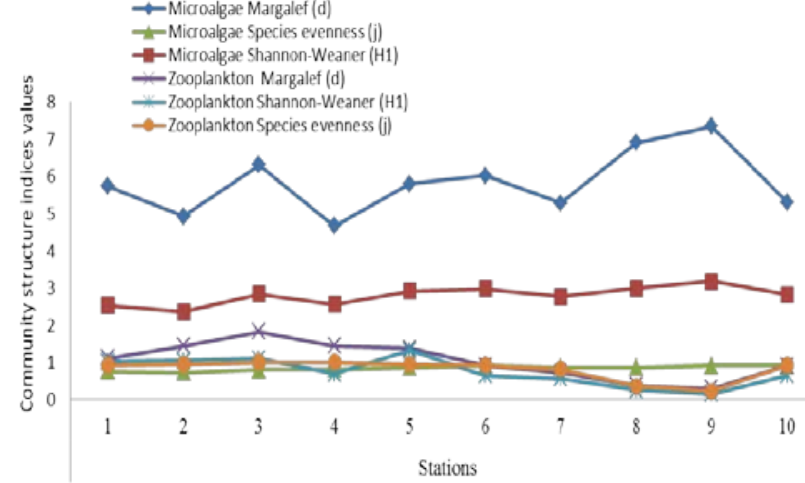

Fig. 3. Comparison of plankton community structure indices at Ibefun River, Ogun State

for $42.2 \%$, while Nais spp. and Perna perna were the least represented, with $9.4 \%$ each of the total individuals $(64$ individuals $/ \mathrm{m}^{2}$ ) recorded (Fig. 5). The general diversity $(\mathrm{H})$ was the highest at Station $1(0.52)$, while the least value $(0.18)$ was recorded at Station 2. The species richness (d) and evenness $(j)$ highest values (1.45 and 1.00) were recorded at Station 8, while Station 2 recorded the lowest values $(0.52$ and 0.60 ) respectively (Fig. 6).
Fish and Fisheries study at Ibefun stations, Ogun state

A total of seventeen (17) finfish and shellfish species comprised of thirteen (13) finfish and four (4) shellfish species were recorded. The finfish and shellfish recorded are representative of freshwater and brackish water species, as shown in Table 7. The dominant species observed were Clarias anguillaris (Clariidae), Tilapia zilli (Cichlidae), Chrysichthys nigrodigitatus (Bagridae), Alestes spp. and Macrobrachium vollenhovenii (Palaemonidae) among others. Condition factor $(\mathrm{k})$ which is an index of condition and degree of wellbeing of the fish varied by size for fish species recorded at the study area, with values that ranged from 1.17-2.96 (Table 7).

\section{Discussions}

Information on variation in physico-chemical factors of the studied area, especially within Ibefun River, were in agreement with earlier observations made in the Lagos lagoon that two dominant factors, fresh water discharge and tidal sea water incursions govern the physical, chemical and biological characteristics of the areas (Nwankwo, 1984, 1996; Brown, 2000). 
252

Table 5. The distribution, occurrence and diversity indices of macrobenthic invertebrates' community at Oluwa and Ifara Rivers

\begin{tabular}{lcccccc}
\hline \multirow{2}{*}{$\begin{array}{l}\text { Macrobenthic } \\
\text { invertebrate }\end{array}$} & \multicolumn{3}{c}{ Oluwa River } & \multicolumn{3}{c}{ Ifara River } \\
\cline { 2 - 7 } & \multicolumn{3}{c}{ Stations } & \multicolumn{3}{c}{ Stations } \\
\cline { 2 - 7 } Hirudinea & 1 & 1 & 2 & - & - & 1 \\
$\begin{array}{l}\text { Glossiphonia spp. } \\
\text { Insecta }\end{array}$ & 1 & & & & & \\
Chironomus spp. & - & 2 & - & 1 & 1 & 2 \\
Oligochaeta & & & & & & \\
Lumbriculus spp. & 2 & 2 & 1 & 1 & 1 & - \\
Number of species $(\mathrm{s})$ & 2 & 3 & 2 & 2 & 2 & 2 \\
$\begin{array}{l}\text { Number of individual } \\
\text { (N) }\end{array}$ & 3 & 5 & 3 & 2 & 2 & 3 \\
$\begin{array}{l}\text { Margalef index }(\mathrm{d}) \\
\text { Shannon-Weaner }\left(\mathrm{H}^{1}\right)\end{array}$ & 0.91 & 1.24 & 0.91 & 1.44 & 1.44 & 0.91 \\
Species evenness $(\mathrm{j})$ & 0.91 & 0.96 & 0.91 & 1.00 & 1.00 & 0.91 \\
\hline
\end{tabular}

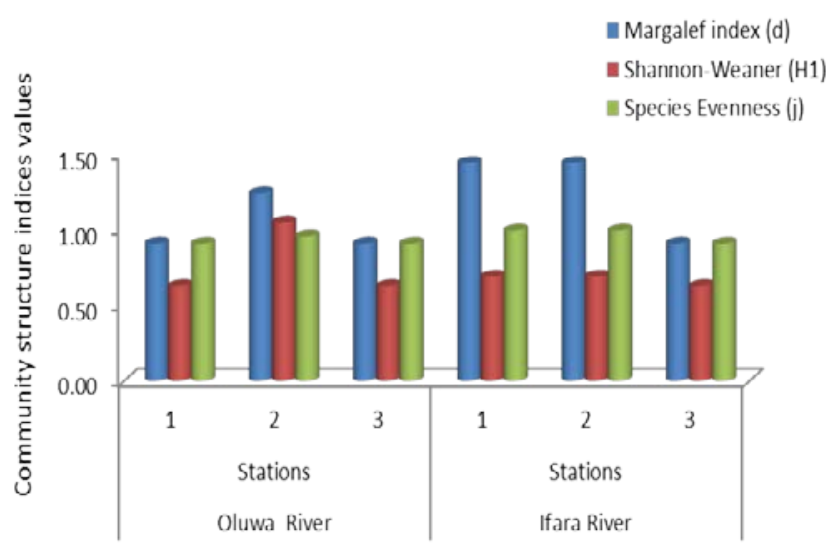

Fig. 4. Comparison of macrobenthic invertebrate community structure indices at Oluwa and Ifara Rivers, Ondo State

Table 6. The distribution, occurrence and diversity indices of macrobenthic invertebrate community at Ibefun River study stations

\begin{tabular}{|c|c|c|c|c|c|c|c|c|c|c|}
\hline \multirow{2}{*}{$\begin{array}{l}\text { Macrobenthic } \\
\text { invertebrate }\end{array}$} & \multicolumn{10}{|c|}{ Stations } \\
\hline & 1 & 2 & 3 & 4 & 5 & 6 & 7 & 8 & 9 & 10 \\
\hline \multicolumn{11}{|l|}{ Oligochaeta } \\
\hline Lumbriculus spp. & 3 & & 4 & & & & 2 & & 2 & \\
\hline Nais spp. & & & 2 & & & 1 & & 1 & & 2 \\
\hline \multicolumn{11}{|l|}{ Bivalvia } \\
\hline Pernaperna (L) & 2 & 1 & & & 1 & 2 & & & & \\
\hline Macoma cumana (C) & 8 & 6 & 1 & 3 & 4 & & 2 & & & 3 \\
\hline \multicolumn{11}{|l|}{ Insecta } \\
\hline Chironomus spp. & 2 & & & 1 & 2 & & 7 & 1 & 1 & \\
\hline Number of taxa & 4 & 2 & 3 & 2 & 3 & 2 & 3 & 2 & 2 & 2 \\
\hline Number of individual & 15 & 7 & 7 & 4 & 7 & 3 & 11 & 2 & 3 & 5 \\
\hline General diversity $(\mathrm{H})$ & 0.52 & 0.18 & 0.41 & 0.24 & 0.42 & 0.28 & 0.39 & 0.30 & 0.28 & 0.29 \\
\hline Maximum diversity (Hmax) & 0.60 & 0.30 & 0.48 & 0.30 & 0.48 & 0.30 & 0.48 & 0.30 & 0.30 & 0.30 \\
\hline Species richness $(\mathrm{d})$ & 1.11 & 0.52 & 1.03 & 0.72 & 1.03 & 0.91 & 0.83 & 1.45 & 0.91 & 0.62 \\
\hline Species evenness $(\mathrm{j})$ & 0.87 & 0.60 & 0.85 & 0.80 & 0.88 & 0.93 & 0.81 & 1.00 & 0.93 & 0.97 \\
\hline
\end{tabular}

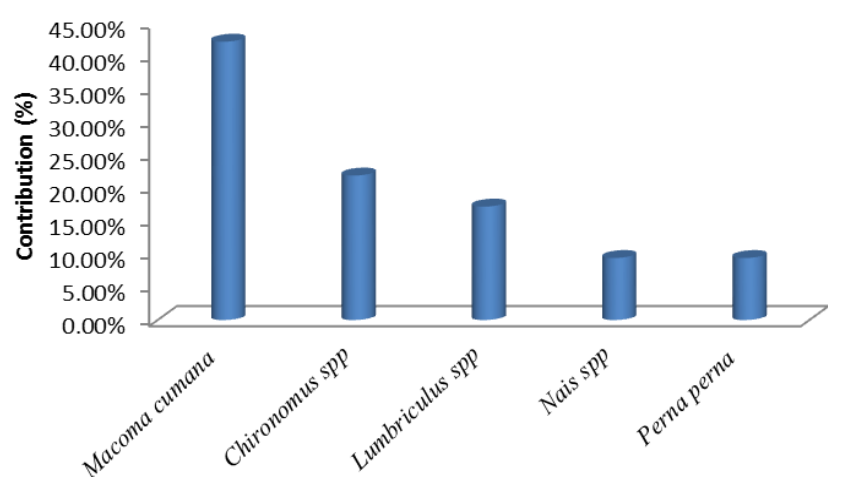

Fig. 5. The percentage contribution of the major macrobenthic invertebrates' taxa at Ibefun, Ogun state

The presence and dominance of diatoms in the sampling areas conformed with observation made by Nwadiaro (1990) in the chanomi creek system of the Niger Delta, Chindah and Pudo (1991) in Bonny River, Erondu and Chindah (1991) in the new Calabar River, Niger Delta, Adesalu and Nwankwo (2005, 2008) in Olero and Abule-Eledu creek respectively, Adesalu et al. (2008) in Ogbe creek and Adesalu and Kunrunmi (2012) in the Lagoons of South-Western Nigeria.

The presence and abundance of Oscillatoria sp. among the blue-green algae supported Nwankwo (2004)

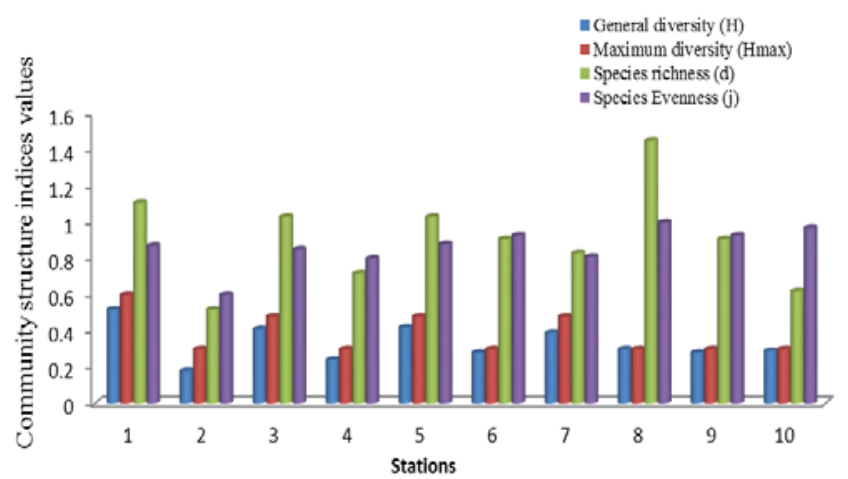

Fig. 6. Comparison of macrobenthic invertebrates community structure indices at Ibefun River, Ogun State

who stated that the blue green algal forms found in the creek were mostly filamentous forms and could be opportunistic forms which by bio-modification of physical processes usually proliferate to advantages of other species.

The dominant of rotifer species in rivers comparative to the cladocerans and copepods is characteristic of tropical lakes and rivers and this has been reported in the studies of Akinbuwa and Adeniyi (1996), Neves et al. (2003), Mwebaza-Nadwula et al. (2005), Imoobe and Akoma (2009). 
Table 7. The checklist and condition factor $(\mathrm{K})$ of fish species at Ibefun River, Ogun state

\begin{tabular}{|c|c|c|c|c|c|}
\hline & Family & Species name & Standard length $(\mathrm{cm})$ & Weight $(\mathrm{g})$ & $\mathrm{K}$-value \\
\hline 1 & Channidae & Parachanna obscura & 25.5 & 225.3 & 1.36 \\
\hline 2 & Clariidae & Clarias anguillaris & 26.3 & 250.2 & 1.38 \\
\hline 3 & Bagridae & Chrysichthys nigrodigitatus (Lacepede) & 28.9 & 295.3 & 1.22 \\
\hline \multirow{3}{*}{4} & \multirow{3}{*}{ Cichlidae } & Tilapia guineensi (Bleeke) & 12.6 & 67.2 & 1.5 \\
\hline & & Tilapia zilli & 16.0 & 105.4 & 2.57 \\
\hline & & Oreochromis aureus & 22.7 & 260.3 & 2.23 \\
\hline 5 & Mormyridae & Hyperopisus bebe & 38.0 & 710.1 & 1.29 \\
\hline 6 & Sphyranae & Sphyraena sphyraena & 44.0 & 998.4 & 1.17 \\
\hline 7 & Cyprinidae & Labeo coubie & 12.0 & 48.2 & 2.79 \\
\hline 8 & Palaemonidae & Macrobrachium vollenhovenii (Herklots) & 6.0 & 6.4 & 2.96 \\
\hline 9 & Ocypodidae & Ocypoda africana & NS & NS & NS \\
\hline 10 & Gecarcinidae & Cardiosoma armatum & NS & NS & NS \\
\hline \multirow{2}{*}{11} & \multirow{2}{*}{ Characidae } & Alestes macrolepidotus (Valenciennes) & 16.5 & 85.1 & 1.89 \\
\hline & & Alestes longipinni (Hubrecht) & 8.2 & 45.2 & 1.00 \\
\hline 12 & Hepsetidae & Hepsetus odoe & 14.7 & 56.3 & 1.25 \\
\hline 13 & Citharinidae & Citharinus citharus (Geoffrey Saint Hilaire) & 10.0 & 47.3 & 1.05 \\
\hline 14 & Clupeidae & Pellonula afzeliusi(Johnels) & 17.0 & 59.1 & 1.32 \\
\hline
\end{tabular}

NS: Not sampled for K-factor analysis

The abundance and distribution of benthic fauna in a given community is affected by the water quality conditions (Bunn and Davies, 1992; Camargo, 1992). Lumbriculidae and Chironomidae have been reported to dominate aquatic benthic invertebrates' communities (Victor and Ogbeibu, 1985; Hynes, 1998) because they hardly show any habitat restriction (Victor and Ogbeibu, 1991) and are known to replace other members of invertebrate community in water bodies under human stressors.

The high load of organic matter in the sediment at the study sites was probably responsible for the prevalence of the bivalves, oligochaetes and the chironomids recorded at some stations. The dominance of bivalve, especially Macoma cumana at Ibefun stations, could be attributed to the suspended small food particles, that seldom settle out of organic matter deposit, which are trapped from the sediment with their feeding apparatus (Yankson and Kendall, 2001). According to Awachie (1981) chironomids usually have no habitat restrictions, while Edokpayi and Osimen (2001) stated that they are known to replace other invertebrate taxa in water bodies polluted by agricultural and domestic activities. Lumbriculus spp. and Nais spp. have been reported in polluted tropical waters (Victor and Ogbeibu, 1985; Atobatele et al., 2005) and prefer soft sediments rich in organic content (Crothers, 1997; Edokpayi et al., 2004), a feature characteristic of the study sites.

The relatively low diversity of benthic macroinvertebrates recorded in this study could be attributed to the resultant effect of anthropogenic impacts on the studied environment (Edokpayi et. al., 2010). However, one of the most important effects of anthropogenic activities such as motorcycle washing, laundering of clothes, farming land nearby, water transportation and sand mining which are prevalent at Ibefun sites were stated by Ogbeibu and Victor (1989) and Edokpayi et al. (2004) as the resultant destabilization of the substratum and increased silt formation in the environment.
The general composition of fish species recorded was similar to those reported for the freshwater and brackish water of West Africa (Schneider, 1990; Holden and Reed, 1991; Olaosebikan and Raji, 1998) probably an indicator of sea water incursion at Ibefun River. The overall community structure at the study sites stretch exhibited low taxa richness. Determining the precise contribution of each factor influencing the abundance of a particular taxon is difficult (Atobatele et al., 2005).

\section{Conclusions}

The low diversity in the zooplankton identified from Oluwa, Ifara and Ibefun Rivers was a clear indication of unstable aquatic communities with negative effect on the aquatic fauna. Among zooplankton, the rotifers were the most diverse, while copepods were the most evenly distributed. Despite the greatest diversity exhibited by diatoms, the euglenoids were the most evenly distributed. According to Lagler (1986), a fish is said to be in good condition when the value of $\mathrm{K}$ is higher than one, therefore the fishes from the water in the study areas were healthy, as indicated by the condition factors observed in the study.

\section{Acknowledgements}

The lead author is grateful to the anonymous environmentalist for providing the logistics.

\section{References}

Adesalu TA, Nwankwo DI (2005). Studies on the Phytoplankton of Olero creek and parts of Benin River, Nigeria. The Ecologia 3(2):21-30.

Adesalu TA, Kunrunmi O (2012). Effects of physico-chemical parameters on phytoplankton of a tidal creek, Lagos, Nigeria Journal of Environment and Ecology 3(1):116-136.

Adesalu TA, Nwankwo DI (2008). Effect of water quality indices on phytoplankton of a sluggish tidal creek in Lagos, Nigeria. Pakistan Journal of Biological Science 11(6):836-844. 
254

Adesalu TA, Abiola TO , Bofia TO (2008). Studies on the epiphytic algae associated with two floating aquatic macrophytes in a sluggish non-tidal polluted creek in Lagos, Nigeria. Asian Journal on Scientific Research 1(4):363-373.

Ajao EA, Fagade SO (1990). Production and population dynamics of Pachymelania aurita Müller. Hydrobiologia 120:97-109.

Akinbuwa O, Adeniyi IF (1996). Seasonal variation, distribution and interrelationships of rotifers in Opa Reservoir, Nigeria. African Journal of Ecology 34:351-363.

Atobatele OE, Morenikeji OA, Ugwumba OA (2005). Spatial variation in physical and chemical parameters and benthic macroinvertebrate fauna of River Ogunpa, Ibadan. The Zoologist 3:58-67.

Awachie JBE (1981). Running water ecology in Africa. In: Lock MA, Williams DD (Eds) Perspectives in running water ecology. Plenum Press, New York and London pp 339-366.

Bannister JV (1976). The length-weight relationship, condition factor and gut contents of the dolphin-fish Coryphaena hippurus (L.) in the Mediterranean. Journal of Fish Biology 9:335-338.

Boney AD (1989). Phytoplankton (2nd edn).

Brown CA (2000).The diversity and density of macrobenthic fauna in the western part of the Lagos lagoon, Lagos, South-West, Nigeria. PhD Thesis, University of Lagos.

Bunn SE, Davies PM (1992). Community structure of macroinvertebrate fauna and water quality of a saline river system in south-western Australia. Hydrobiologia 248:143-160.

Camargo JA (1992). Macroinvertebrate responses along the recovery gradient of a regulated river (Spain) receiving an industrial effluent. Archives of Environmental Contamination and Toxicology 23:324-332.

Castro P, Huber ME (2005). Marine biology (3rd ed). McGraw Hill Higher Education, New York, USA.

Chindah AC, Pudo J (1991). A preliminary checklist of algae found in plankton of Bonny River in Niger Delta, Nigeria. Fragmenta Floristica et Geobotanica 36(1):112-126.

Crothers J (1997). A key to the major groups of British marine invertebrates. Field Studies 9(1):1-177.

Davis CC (1955). The marine and freshwater plankton. Michigan State University Press, Michigan pp 562.

Desikachary TV (1959). Cyanophyta. Indian Council of Agriculture Research, New Delhi.

Edokpayi CA, Osimen CA (2001). Hydrobiological studies on Ibiekuma River at Ekpoma, southern Nigeria, after impoundment: the faunal characteristics. African Journal of Science and Technology 2(1):72-81.

Edokpayi CA, Lawal MO, Okwok NA, Ogunwenmo CA (2004). Physico-chemical and macrobenthic faunal characteristics of Kuramo water, Lagos, Southern Nigeria. African Journal of Aquatic Science 29(2):235-241.

Edokpayi CA, Olowoporoku AO, Uwadiae RE (2010). The hydrochemistry and macrobenthic fauna characteristics of an urban draining creek. International Journal of Biodiversity and Conservation 2(8):196-203.

Erondu ES, Chindah AC (1991) Physico-chemical phytoplankton changes in a tidal freshwater station of the New Calabar River South Eastern Nigeria. Environment and Ecology 9(3):561-570.
Haslam SM (1990). River pollution. An Ecological Perspective. Belhaven Press.

Holden M, Reed W (1991). West Africa fresh water fishes. Longman Group Ltd, Harlow, UK.

Hynes KE (1998). Benthic macroinvertebrate diversity and biotic indices for monitoring of 5 urban and urbanizing lakes within the Halifax Regional Municipality (HRM), Nova Scotia, Canada. Soil \&Water Conservation Society of Metro Halifax 14:114.

Ibemenuga KN, Inyang N (2006). Macroinvertebrate fauna of a tropical freshwater stream in Nigeria. Animal Research International 3(3):553-561.

Ikomi RB, Arimoro FO, Odihirin OK (2005). Composition, distribution and abundance of macroinvertebrates of the upper reaches of river Ethiope, Delta State, Nigeria. The Zoologist 3:6881.

Imoobe TOT, Akoma OC (2009). Spatial variations in the composition and abundance of zooplankton in the Bahir Dar Gulf of Lake Tana. Ethiopia African Journal of Ecology 48:72-77.

Krammer H, Large-Bertalot H (1986). Bacillariophyceae. 1. Teil: Naviculaceae In: Ettl H, GerloffJ, Heynig H, Mollenhauer D (Eds). Süsswasserflora von Mitteleuropa, Fischer Verlag, Stuttgart.

Lagler KF (1986). Ichthyology.John Wiley and Sons, London.

Margalef DR (1951). Diversidad de especies en les communideades natural. Public Institute of Biologic Barcelonia 9:5-27.

Mwebaza-Nadwula L, Sekiranda SBK, Kiggundu V (2005). Variability in zooplankton Community along a section of the Upper Victoria Nile, Uganda. African Journal of Ecology 43:251-257.

Neves IF, Rocha O, Roche KF, Pinto AA (2003). Zooplankton community structure of two marginal lakes of the river Cuibá (MatoGrosso, Brazil) with analysis of Rotifera and Cladocera diversity. Brazilian Journal of Biology 63:329-343.

Nkwoji JA, Yakub A, Ajani GE, Balogun KJ, Renner KO, Igbo JK, Ariyo AA, Bello BO (2010). Seasonal variations in the water chemistry and benthic macroinvertebrates of a south western Lagoon, Lagos, Nigeria. Journal of American Science 6(3):85-92.

Nwadiaro CS (1990). A hydrobiological survey of the Chanomi creek system, lower Niger Delta, Nigeria. Limnologica 21:263-274.

Nwankwo DI (1984). Seasonal changes of phytoplankton of Lagos lagoon and the adjacent sea in relation to environmental factors. PhD Thesis, University of Lagos.

Nwankwo DI (1996). Phytoplankton diversity and succession in Lagos Lagoon, Nigeria. Archiv für Hydrobiologie 135:529-678.

Nwankwo DI (2004). Studies on the environmental reference of bluegreen algae (cyanophyta) in Nigeria coastal waters. The Nigeria Environmental Society Journal 2(1):44-51.

Odiete WO(1999). Environmental physiology of animals and pollution. Diversified Resources Lagos, Nigeria.

Ogbeibu AE (2005). Biostatistics: A practical approach to research and data handling. Mindex Publishing Company limited, Benin, Nigeria.

Ogbeibu AE, Victor R (1989). The effect of road and bridge construction on the bank-root macrobenthic invertebrates of a southern Nigerian stream. Environmental Pollution 56:85-100.

Olaniyan CIO (1975). An introduction to West African ecology. Heinemann Education Books Ltd., London. 
Olaosebikan BD, Raji A (1998). Field guide to Nigerian freshwater fishes. Federal College of Freshwater. Fisheries Technology, New Bussa, Nigeria.

Patrick R, Reimer CW (1966). The diatoms of the United States. Academy of Natural Sciences.

Patrick RM, Reimer CW (1975). The diatoms of the United States, exclusive of Alasuka and Hawaii. Academy of Natural Sciences of Philadelphia.

Pielou EC (1975). The measurement of diversity to different types of biological collections Journal of Theoretical Biology 13:131-144.

Prescott GW (1961). Algae of the Western Great Lake area. Dubuque. WC Brown Company.

Quigley M (1977). Invertebrates of streams and rivers-a key to identification. Edward Arnold (Publishers) Ltd, London.

Ravera O (2000). Ecological monitoring for water body management. Proceedings of monitoring Tailor made III. International Workshop on Information for Sustainable Water Management pp:157-167.

Schneider W (1990). Field guide to the commercial marine resources of the Gulf of Guinea, FAO.
Shannon CE, Weaver W (1963). The mathematical theory of communication. University of Illinois Press, Urbana, Illinois.

Victor R, Ogbeibu AE (1985). Macrobenthic invertebrates of a stream flowing through farmlands in southern Nigeria. Environmental Pollution Series A 39:337-349.

Victor R, Ogbeibu AE (1991). Macroinvertebrate communities in the erosional biotope of an urban stream in Nigeria. Tropical Zoology 4:1-12.

Whitford LA, Schumacher GJ (1973). A manual of fresh water algae. Raleigh, North Carolina.

Wiafe G, Frid CLJ (2001). Marine zooplankton of West Africa. Darwin Initiative Report.

Wimpenny RS (1966). The plankton of the sea. Faber and Faber Limited, London, England.

Yankson K, Kendall M (2001). A student's guide to the seashore of West Africa. Darwin Initiative Report 1, Ref 162/7/451 pp 132. 